\title{
Impact of DDDAS/InfoSymbiotics in the Industrial Sector
}

\author{
Daniel Abramovitch ${ }^{1}$, Sandeep Gogineni ${ }^{2}$, Cory Kays ${ }^{3(\otimes)}$, Chung-Sheng Li $^{4}$, \\ Jose Moreira $^{5}$, Chitra Sivanandam ${ }^{6}$, and Nurali Virani ${ }^{7}$ \\ 1 Agilent Technologies, Santa Clara, CA 95051, USA \\ 2 Information Systems Laboratories, La Jolla, CA 92037, USA \\ 3 Cornerstone Research, San Francisco, CA 94111, USA \\ kaysca@crgrp.com \\ 4 PriceWaterhouseCooper, New York 10017, USA \\ 5 IBM, TJWatson Research, Yorktown Heights, NY 10598, USA \\ 6 Science Applications International Corporation (SAIC), Reston, VA 20190, USA \\ 7 GE, Boston, MA 02210, USA
}

\begin{abstract}
This panel convenes representatives from several industries in the information technology, aerospace, power, manufacturing and finance sectors, who will address how advances in modeling and prediction methods, and decision support for complex systems, as well as the underlying information technology infrastructures, can advance the capabilities in their respective sectors. Part of the discussion will include research and technology development within their own organizations as well as the broader academic and research laboratories communities, and potential for collaborative and synergistic advances across the industry, academe, and federal sectors can enhance the opportunities for advances and capabilities sought.
\end{abstract}

Keywords: Industry panel $\cdot$ Modeling $\cdot$ Prediction 